\title{
Brief communication: Application of a muonic cosmic ray snow gauge to monitor the snow water equivalent on alpine glaciers
}

\author{
Rebecca Gugerli ${ }^{1}$, Darin Desilets ${ }^{2}$, and Nadine Salzmann ${ }^{1}$ \\ ${ }^{1}$ Department of Geosciences, University of Fribourg, Switzerland \\ ${ }^{2}$ Hydroinnova LLC, Albuquerque, USA
}

Correspondence: Rebecca Gugerli (rebecca.gugerli@unifr.ch)

\begin{abstract}
Monitoring the snow water equivalent (SWE) in the harsh environments of high mountain regions is a challenge. Here, we explore the use of muon counts to infer SWE. We deployed a muonic cosmic ray snow gauge ( $\mu$-CRSG) on a Swiss glacier during the snow rich winter season 2020/21 (almost $2000 \mathrm{~mm}$ w.e.). The $\mu$-CRSG measurements agree well with measurements by a neutronic cosmic ray snow gauge (n-CRSG) and they lie within the uncertainty of manual observations. We conclude that the $\mu$-CRSG is a highly promising method to monitor SWE in remote high mountain environments with several advantages over the $\mathrm{n}$-CRSG.
\end{abstract}

\section{Introduction}

The snow water equivalent (SWE) of the seasonal snowpack is a key variable of the hydrological and climate system and highly relevant for hydrological, glaciological and meteorological studies, especially in high mountain regions. However, operational monitoring of SWE in high mountain regions still poses considerable technical and logistic challenges because of the harsh environmental conditions (wind, icing, etc.) and the remoteness of the measurement sites (e.g., Kinar and Pomeroy, 2015; Nitu et al., 2018). As a result, temporally continuous and accurate SWE measurements in high mountain regions are very scarce and/or associated with significant uncertainties.

Several investigated methods take advantage of naturally occurring cosmic radiation to infer SWE temporally continuously. These methods make use of naturally occurring gamma radiation (e.g., Osterhuber et al., 1998; Choquette et al., 2008) or of neutrons from secondary cascades of cosmic rays (Kodama et al., 1975). The neutronic cosmic ray snow gauge (n-CRSG), a method proposed by Kodama et al. (1975), measures the attenuation of incoming secondary neutrons to infer SWE. This has proved successful (e.g., Wada et al., 1977; Kodama et al., 1979; Kodama, 1980; Avdyushin et al., 1982), especially for remote and harsh environments (e.g., Howat et al., 2018; Gugerli et al., 2019). Nonetheless, some drawbacks such as the limited measurement precision that can be achieved with a reasonably sized sensor have been identified (e.g., Gugerli et al., 2019).

A similar cosmic ray method measures neutrons scattered near the land-atmosphere boundary with an above-ground n-CRSG (e.g., Desilets et al., 2010; Rasmussen et al., 2012). The great advantage is that it is non invasive and offers a large footprint. However, it is limited to SWE amounts of around $600 \mathrm{~mm}$ w.e. in non-glacierized areas (Schattan et al., 2017).

Instead of using secondary neutrons as outlined above, we here investigate a muonic cosmic ray snow gauge ( $\mu$-CRSG) to 
obtain temporally continuous SWE measurements. Cosmic ray muons are highly penetrating particles and thus not as sensitive to SWE as neutrons. But, the highly penetrating nature of muons also makes them far more abundant than neutrons at ground level, and provides a compensating statistical advantage over neutrons that should result in a better measurement precision. However, unlike neutrons, muons are unstable and can decay in mid flight. For this reason, muon intensity at ground level is influenced by the distance traveled, or, more specifically, by the thickness of the atmosphere on any given day (e.g., Riádigos et al., 2020). Hence, there are several known and probably also unknown trade offs between neutrons and muons with consequences on inferring SWE from these measurements.

The aim of this study is to explore the use of muons to infer temporally continuous SWE in a high mountain glacierized site, and to provide a first-cut calibration function for the $\mu$-CRSG. The $\mu$-CRSG measurements are compared to manually obtained SWE and to hourly SWE measurements obtained by a n-CRSG. Furthermore, we discuss the advantages of a $\mu$-CRSG with a focus on SWE monitoring in remote and harsh high mountain environments.

\section{Study site and data}

\subsection{Study site}

In December 2020, we deployed two $\mu$-CRSG (prototype Bruno provided by Hydroinnova LLC) on the Glacier de la Plaine Morte in the Swiss Alps. The glacier has an area of $7.1 \mathrm{~km}^{2}$ (2019) and elevation bands from $2650 \mathrm{~m}$ a.s.l. to $2800 \mathrm{~m}$ a.s.l. It

40 is the largest plateau glacier of the European Alps (GLAMOS, 2020).

An automatic weather station with a n-CRSG (SnowFox ${ }^{\mathrm{TM}}$ provided by Hydroinnova LLC) was deployed in autumn 2016 on Plaine Morte ( $46^{\circ} 22.8^{\prime} \mathrm{N}, 7^{\circ} 29.7^{\prime} \mathrm{E}, 2689 \mathrm{~m}$ a.s.l., see Gugerli et al., 2019, for more information). The two $\mu$-CRSG were added to this station, one buried below the snow, i.e. lying on the glacier ice surface close to the n-CRSG, and one added at the top of the station at $4.8 \mathrm{~m}$ height above the glacier ice surface. While only one n-CRSG is deployed, two $\mu$-CRSG are necessary to account for atmospheric influences on the muon count rates. For the n-CRSG, parameterizations have previously been investigated to correct for changes in atmospheric pressure and incoming cosmic ray fluxes.

\subsection{Data}

This study encompasses three types of observational data sets. First, five manual SWE measurements were obtained between 16 December 2020 and 20 May 2021 by means of snowpits and snow cores, which complement a series of totally 22 manual SWE measurements between 20 Oct 2016 and 20 May 2021 at the same site (Gugerli, 2021). The uncertainty of these manual observations is defined as the standard deviation of several observations during the same field day. Second, hourly SWE obtained by a n-CRSG are available from 20 Oct 2016 to 13 August 2021 (Gugerli et al., 2019) and validated with the 22 manual SWE measurements. Third, two $\mu$-CRSG were deployed on 16 December 2020 and provided hourly measurements until 13 August 2021.

55 From 16 December 2020 to 13 August 2021, 241 days of hourly neutron counts and 213 days of hourly muon counts were 
https://doi.org/10.5194/tc-2021-277

Preprint. Discussion started: 7 September 2021

(c) Author(s) 2021. CC BY 4.0 License.

(c) (i)

obtained. The data gaps within the muon count rates are due to unusual amounts of snow in the winter season 2020/21, which buried the solar panels and interrupted power supply. Since the solar panels of the n-CRSG are mounted higher up, and the measurement setup contains larger batteries, these measurements were not interrupted by the large snow amounts.

\section{Methods}

60 To assess the performance of the $\mu$-CRSG, we (i) process neutron and muon counts to make them directly comparable and (ii) compare SWE inferred from neutron and muon counts over time. While the n-CRSG is an established method and conversion functions have been thoroughly assessed, using $\mu$-CRSG is a novel approach and no data or conversion functions exist so far. Hence, we derive a conversion function based on our manually obtained SWE observations.

\subsection{Neutronic cosmic ray snow gauge (n-CRSG)}

\subsubsection{Correcting neutron counts}

The hourly neutron counts of the n-CRSG are first corrected for influences from incoming neutron fluxes and for variations in barometric air pressure. Following today's standard correction functions for sub-snow n-CRSG (e.g., Howat et al., 2018; Gugerli et al., 2019), we use hourly in situ pressure measurements ( $p_{i}$ in $\mathrm{hPa}$ ), the attenuation length at the site $(L=132 \mathrm{hPa})$ and hourly neutron count rates ( $F_{\mathrm{inc}, i}$ in cps) from a reference neutron monitor located on Jungfraujoch in Switzerland (JUNG, http://www.nmdb.eu/) with a site-specific adjustment factor for Plaine Morte $(\beta=0.95)$. The corrected hourly neutron counts ( $N_{\text {corr }, i}$ in cph) are obtained as

$N_{\text {corr }, i}=N_{\text {raw }, i} \cdot\left(\beta \cdot\left(\frac{F_{\text {inc }, i}}{F_{\text {inc }, 0}}-1\right)+1\right) \cdot \exp \left(\frac{p_{i}-p_{0}}{L}\right)$.

The reference values for $F_{\text {inc, }, 0}(\mathrm{cph})$ and $p_{0}(\mathrm{hPa})$ correspond to the $24 \mathrm{~h}$ mean from 12 July 201708 UTC to 13 July 201708 UTC.

\subsubsection{Inferring SWE from neutron counts}

The corrected neutron counts $\left(N_{\mathrm{corr}, i}\right)$ are converted to hourly SWE $\left(S W E_{\mathrm{n}, i}\right.$ in cm w.e.) by

$S W E_{\mathrm{n}, i}=-\frac{1}{\Lambda_{i}} \cdot \ln \frac{N_{\mathrm{corr}, i}}{N_{0}}$

where the variable $\Lambda_{i}$ is the effective attenuation length given by

$\Lambda_{i}=\frac{1}{\Lambda_{\max }}+\left(\frac{1}{\Lambda_{\min }}-\frac{1}{\Lambda_{\max }}\right) \cdot\left(1+\exp \left(-\frac{\frac{N_{\text {corr }, i}}{N_{0}}-a_{1}}{a_{2}}\right)\right)^{-a_{3}}$

80 The snow free count rate $\left(N_{0}\right.$ in $\left.\mathrm{cph}\right)$ corresponds to the median of the corrected neutron counts $\left(N_{0}=4146 \mathrm{cph}\right)$ during the same 24h reference period used for the correction factors (12 July 201708 UTC to 13 July 201708 UTC). The unitless calibration 
https://doi.org/10.5194/tc-2021-277

Preprint. Discussion started: 7 September 2021

(c) Author(s) 2021. CC BY 4.0 License.

(c) (1)

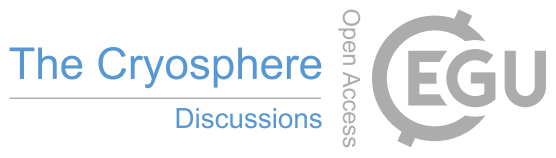

factors $a_{1}, a_{2}, a_{3}$ are $0.31,0.08$ and 1.12, respectively. The attenuation lengths $\Lambda_{\max }$ and $\Lambda_{\min }$ are $114.4 \mathrm{~cm}$ and $14.1 \mathrm{~cm}$, respectively (Howat et al., 2018; Gugerli et al., 2019).

To increase our confidence in the n-CRSG observations, we extend the previous validation of the n-CRSG on Plaine Morte from nine (Gugerli et al., 2019) to 22 manually obtained SWE estimates by snow pits and snow cores (Fig. 1). The 22 manual measurements are significantly and highly correlated with a coefficient of determination of 0.969 (Fig. 1a). On average, the n-CRSG agrees with the manually obtained SWE with an underestimation of $-1 \%$ and an uncertainty of $\pm 12 \%$ (one standard deviation, Fig. 1b).
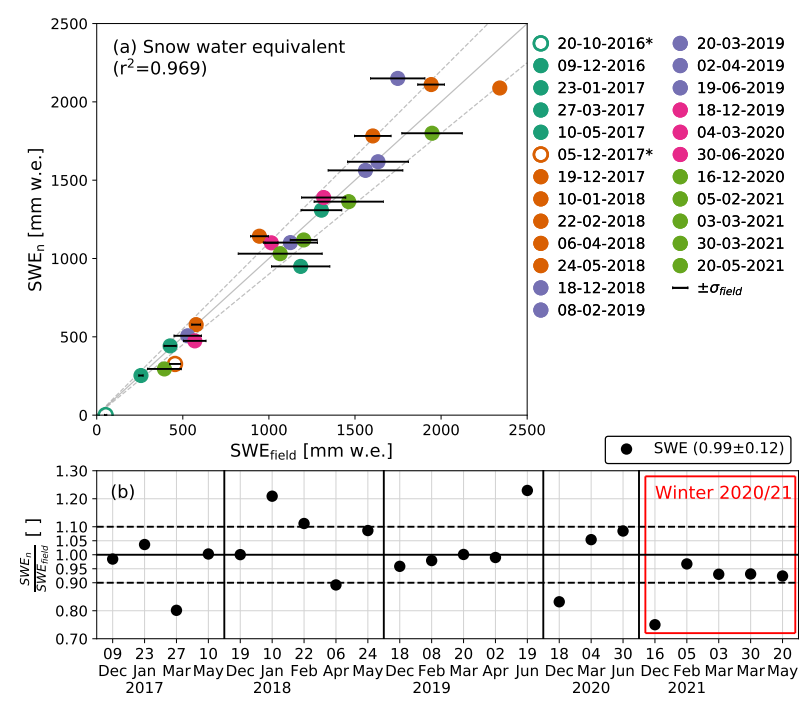

Figure 1. Validation of the n-CRSG. Panel (a) shows SWE derived by the $n$-CRSG compared to SWE derived manually with a $r^{2}$ of 0.969 . The field observations from 20 Oct 2016 and 5 Dec 2017 were done while deploying the devices and are thus not taken into account for the validation. [Figure adapted from Gugerli (2021)]

\subsection{Muonic cosmic ray snow gauge ( $\mu$-CRSG)}

90 We use two $\mu$-CRSG deployed on the glacier site; one below and one above the snowpack. Monitoring the incoming muon counts with the sensor above the snowpack allows to directly correct for the temporal variability caused by atmospheric effects such as air pressure variations and variations in incoming cosmic ray fluxes. Besides these effects, the muon intensity also depends on the temperature profile of the atmosphere. The temperature influences the production rate of muons (positive temperature effect) as well as its decay rate (negative temperature effect, e.g., de Mendonça et al., 2016; Riádigos et al., 2020). 
https://doi.org/10.5194/tc-2021-277

Preprint. Discussion started: 7 September 2021

(c) Author(s) 2021. CC BY 4.0 License.

(c) (i)

\subsubsection{Correcting muon counts}

To obtain a time series of muon count rates corrected for atmospheric influences, we multiply the count rate under snow free conditions with the relative muon count rate $\left(f_{\mu, i}\right)$. The relative count rate is derived as

$f_{\mu, i}=\frac{\mu_{\mathrm{sub}, i}}{\mu_{\mathrm{top}, i}}$

where $\mu_{\mathrm{top}, i}\left(\mu_{\mathrm{sub}, i}\right)$ is the hourly count rate of the $\mu$-CRSG above (below) the snowpack. We assume that atmospheric influences are manifested in the measurements of both devices and that the relative count rate only represents changes related to the snowpack (accumulation and ablation). With the relative count rate, we can derive the corrected muon count rate $\left(\mu_{\text {corr }, i}\right.$ in $\mathrm{cph})$ as

$\mu_{\mathrm{corr}, i}=\mu_{\mathrm{sub}, 0} \cdot f_{\mu, i}$.

The variable $\mu_{\text {sub }, 0}$ corresponds to the mean daily count rate under snow free conditions on the 12 August 2021 from 0 UTC until $23 \mathrm{UTC}$ ( $\mu_{\mathrm{sub}, 0}=42202 \mathrm{cph}$ ). This measurement is obtained from the $\mu$-CRSG lying on the glacier ice surface to assure a direct comparison to the n-CRSG, which is also lying on the ice surface.

\subsubsection{Inferring SWE from muon counts}

The conversion function used to infer SWE from muon counts is derived by using the manual field observations on Plaine Morte. Independent data obtained by descending the same prototype of the $\mu$-CRSG into Cochiti lake in New Mexico, USA

110 (1702 m a.s.l., see supplement) exist. However, we cannot use these data directly because of the different locations, and more importantly, the different elevations. Nonetheless, the decreasing muon count rate with water depth suggests a discontinuous function with a transition in slope (muon attenuation lenght) between $1000 \mathrm{~mm}$ and $1500 \mathrm{~mm}$ water depth (see Fig.S1), and we base our assumption of a two-part conversion function on these measurements.

Our conversion function is derived by splitting the five available manual field measurements into two parts. For the first part of the discontinuous function, we use the observations from 16 Dec 2020 and 5 Feb 2021, where we fit an exponential function. The second part of the conversion function is obtained through a fit between the manual observations on 5 Feb 2021 and 20 Mai 2021. The discontinuous function transitions at the SWE amount obtained on $5 \mathrm{Feb} 2021$, which corresponds to a relative muon count rate of 0.65 . This yields

$$
\begin{gathered}
\text { if } \frac{\mu_{\mathrm{sub}, i}}{\mu_{\mathrm{top}, i}}<=0.65 \quad S W E_{\mu, i}=-265 \cdot \ln \left(\frac{\mu_{\mathrm{sub}, i}}{\mu_{\mathrm{top}, i}}\right)-7 \\
\text { if } \frac{\mu_{\mathrm{sub}, i}}{\mu_{\mathrm{top}, i}}>0.65 \quad S W E_{\mu, i}=-538 \cdot \ln \left(\frac{\mu_{\mathrm{sub}, i}}{\mu_{\mathrm{top}, i}}\right)-124
\end{gathered}
$$

to convert relative muon counts to hourly $\operatorname{SWE}\left(S W E_{\mu, i}\right.$ in $\mathrm{mm}$ w.e.). 
https://doi.org/10.5194/tc-2021-277

Preprint. Discussion started: 7 September 2021

(c) Author(s) 2021. CC BY 4.0 License.

(c) (i)

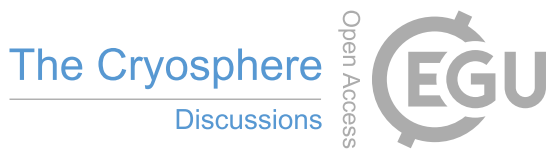

\section{Results and discussion}

\subsection{Comparison of sub-snow neutron and muon counts}

The evolution of neutron and muon counts over the winter season 2020/21 is presented in Fig. 2. When the muon detectors were deployed in December 2020, the snowpack had a depth of $140 \mathrm{~cm}$ with a SWE of $393 \pm 98 \mathrm{~mm}$ w.e. (16 December 2020). Hence, the muon counts above and below the snowpack differ in the beginning of the measurements (Fig. 2b). This difference increases with the deepening snowpack until beginning of June 2021. In June, snow ablation dominates and the difference between the sub and top $\mu$-CRSG decreases until they have similar count rates in August 2021, when the site becomes snow free.

The temporal variability of the corrected muon counts (Fig. 2b) correlate well with the temporal variability in corrected neu-
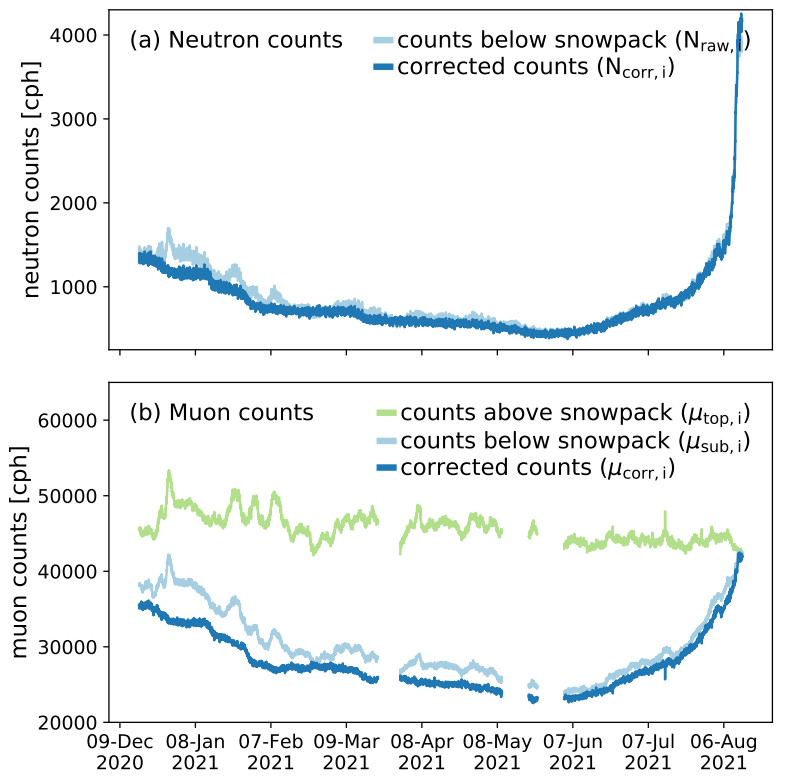

Figure 2. Neutron and muon counts from 16 Dec 2020 to 13 August 2021. Panel (a) shows neutron counts from the n-CRSG and (b) muon counts from the $\mu$-CRSG during the same time period. Please note the different scales for the y-axis.

tron counts (Fig. 2a). As with the n-CRSG, periods with snow accumulation show decreasing counts (e.g., mid January 2021 to beginning of February 2021) and periods with snow ablation increasing counts (e.g., mid June to end of July). In between the count rate remains stable (e.g., end December to mid January). Considering the uncorrected neutron and muon counts, temporal fluctuations related to atmospheric effects are very similar, too (Fig. 2).

Comparing neutron and muon counts, the counting statistics are highly different, which influences the uncertainty of these counts. The uncertainty of the count rate is defined as the square root of the count rate divided by the count rate itself. Gugerli et al. (2019) demonstrate that the main contributor to a low measurement precision of the n-CRSG, especially for deep snow- 
packs, is the uncertainty within the neutron counts. This precision is estimated through error propagation of a non-linear equation considering all variables (see Eq. 1) with their uncertainties (see Table 5 in Gugerli et al., 2019). The corrected neutron counts range between $379 \mathrm{cph}$ and $4256 \mathrm{cph}$, and the corrected muon counts have count rates between $22540 \mathrm{cph}$ and $42479 \mathrm{cph}$. The higher count rate strongly reduces the uncertainty of these measurements. While the uncertainty of the counts range between $1.5 \%$ and $5.1 \%$ for the neutrons, they lie between $0.5 \%$ and $0.7 \%$ for the muons. Note that these uncertainties only refer to the count rates and do not include potential systematic biases or influences by the parameterization of correction functions.

\subsection{Evaluation of SWE inferred by muon counts}

The good agreement in the evolution of neutron and muon count rates presented in Fig. 2 shows the potential for using muon counts to infer SWE. Figure 3a shows an exponential relationship between the muon count rates and SWE at a daily resolution (manually obtained SWE) as well as at an hourly resolution (SWE obtained by the n-CRSG).

As suggested by the independent data obtained in a lake (cf. Sect. 3.2.2 and the supplement), a discontinuity within the relation

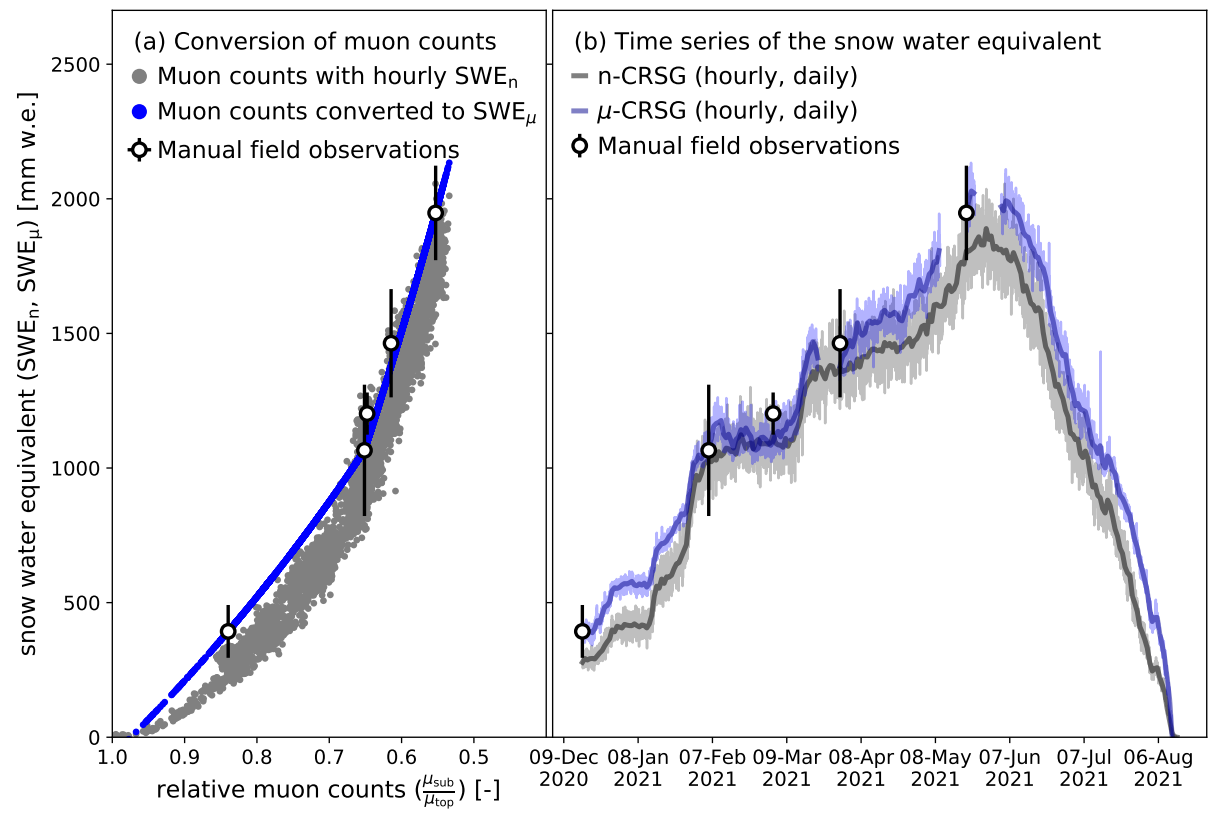

Figure 3. From muon count rates to SWE. Panel (a) shows the relative muon count rates plotted against SWE that is measured by the n-CRSG (grey dots) and measured manually (white dots). Blue dots represent the SWE that is directly inferred from the relative muon count rate with the conversion function given in Eq. 6. Panel (b) shows the time series of SWE inferred from neutron counts (grey) and muon counts (blue) at a daily resolution. Light grey and light blue represent hourly observations of the n-CRSG and $\mu$-CRSG, respectively.

between relative muon counts and SWE is manifested in Fig. 3a with the manually obtained SWE and SWE derived from the 
n-CRSG. Figure 3a indicates a potential transition between $750 \mathrm{~mm}$ w.e. and $1250 \mathrm{~mm}$ w.e., which is in line with the data obtained from lake experiments. Due to the snow rich winter season of 2020/21, only one field measurements (1065 mm w.e., 5 Feb 2021) is available within this transition bin. SWE amounts larger than $1065 \mathrm{~mm}$ w.e. (5 Feb 2021) are better represented. In the conversion function presented here we account for this transition in the attenuation length of muons with increasing SWE. To the best of the authors knowledge, however, no other data is currently available to derive a conversion function that is suitable for our glacierized site. Thus, our conversion function relies on the manual field measurements. While this results in a good agreement between $\mu$-CRSG SWE and n-CRSG SWE, some limitations remain. With the fit between relative muon count rates and manually obtained SWE, the condition of having $0 \mathrm{~mm}$ w.e. for a relative muon count rate of 1.0 is not fulfilled. Either a third part of the conversion needs to be introduced, or the fit needs to be repeated with more manual measurements. In both cases, more data is necessary. Furthermore, a robust statistical evaluation is not possible nor representative because only two manual field measurements remain independent.

Manually obtained SWE observations also carry uncertainties, which are depicted in Fig.3. These correspond to the standard deviation of several measurements obtained close to the sensor and on the same day. Especially measurements within deep snowpacks are laborious and may have significant uncertainties due to limited access to deeper layers. The uncertainty of these field observations vary between 7\% (3 March 2021) and 25\% (16 Dec 2020). The latter was especially challenging to measure because of an unusually deep layer of light and powdery snow.

Despite these uncertainties, we derive temporally continuous SWE from muon counts (Fig. 3b) that agree well with independent hourly SWE measurements by the n-CRSG. Mostly, daily n-CSRG and $\mu$-CRSG lie within the uncertainty of the manual field observations (Fig. 3b). Note that the agreement with some of the manually-obtained field data is related to how the conversion function was derived.

Generally, the hourly SWE by the $\mu$-CRSG has less variability throughout the day than hourly SWE by the n-CRSG. Nonetheless, daily $\mu$-CRSG SWE fluctuates more in February compared to the n-CRSG SWE. Two major Sahara dust events (5-6 Feb 2021 and 22-25 Feb 2021 MeteoSchweiz, 2021) could be related to these fluctuations, but this remains speculative. Apart from this period, the temporal fluctuations are consistent between n-CRSG and $\mu$-CRSG SWE.

\subsection{The potential of the muonic cosmic ray snow gauge to monitor SWE in high mountain regions}

Our results from two $\mu$-CRSG deployed on a glacierized sites confirm the promising approach of using $\mu$-CRSG to infer temporally continuous SWE on glacierized high mountain sites. With the improved counting statistics, the uncertainty of the count numbers is reduced by almost a factor of 10 compared to neutron count uncertainties. As Gugerli et al. (2019) show, the uncertainty in the count numbers are the largest contributor to the overall uncertainty of these measurements. Hence, the $\mu$-CRSG promises to infer sub-daily SWE estimates with a higher precision than the n-CRSG.

Moreover, the $\mu$-CRSG has additional important advantages regarding its suitability and applicability in remote high mountain environments compared to a n-CRSG. The $\mu$-CRSG is technically more robust and lighter, it consumes less energy and is overall cheaper in its production as it does not require exotic fill gases or elaborate cleaning procedures during manufacture. 
https://doi.org/10.5194/tc-2021-277

Preprint. Discussion started: 7 September 2021

(c) Author(s) 2021. CC BY 4.0 License.

(c) (i)

\section{Conclusions and perspectives}

This study presents the potential of monitoring SWE in glacierized high mountain environments by means of muon counts.

We infer SWE from a relative muon count rate from two $\mu$-CRSGs deployed on an alpine glacier. The direct comparison to independent n-CRSG observations demonstrates the proof of concept of inferring SWE from muons and highlights the great potential for glacierized high mountain regions. This study further advances our knowledge and possibilities of monitoring SWE accurately and reliably in technical challenging environments.

The main limitation of our study is the number of manually obtained SWE observations. Due to logistical and financial restrictions no further manual measurements were possible, and to the best of the author's knowledge, no other data for further analysis for the glacierized site are available. This limitation is addressed by including hourly SWE measurements by a nCRSG that have extensively been validated (cf. Sect. 3.1.2).

In future studies, more manual measurements and simulations can improve and validate the conversion function. In addition, correction functions for incoming variations, which are similar to the parameterizations of the n-CRSG, can be derived. Potential correction functions for the temperature effect on the muon intensity have been previously investigated (e.g., Ganeva et al., 2013), and should be analysed for the application of a sub-snow $\mu$-CRSG. Once such influences can be accounted for, only one $\mu$-CRSG deployed below the snowpack would be needed. Being cheaper and lighter than the n-CRSG, more devices can be deployed covering larger areas and thus reducing uncertainties in area-wide SWE by remote sensing and/or modelling approaches.

Data availability. All data will be available in a future repository.

Author contributions. RG conducted the analysis and prepared the manuscript with input from all authors. DD contributed to the data analysis. All authors contributed to the design of this study.

Competing interests. RG and NS declare that they have no competing interests. The author DD is the owner of Hydroinnova LLC. grateful to all field helpers who joined us on Plaine Morte to obtain manual snow observations. We acknowledge the NMDB database (www.nmdb.eu) founded under the European Union's FP7 programme (contract no. 213 007), and the PIs of individual neutron monitors 
https://doi.org/10.5194/tc-2021-277

Preprint. Discussion started: 7 September 2021

(C) Author(s) 2021. CC BY 4.0 License.

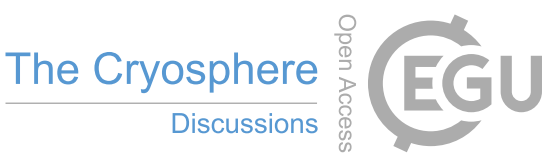

at: IGY Jungfraujoch and NM64 Jungfraujoch (Physikalisches Institut, University of Bern, Switzerland) for the data provided to correct the neutronic cosmic ray snow gauge counts for the incoming cosmic ray flux. 
https://doi.org/10.5194/tc-2021-277

Preprint. Discussion started: 7 September 2021

(c) Author(s) 2021. CC BY 4.0 License.

(c) (i)

\section{References}

Avdyushin, S. I., Kolomeyets, E. V., Nazarov, I. M., Pegoyev, A. N., and Fridman, S. D.: Application of Cosmic Rays To the Solution of Some Hydrological Problems, in: Proceeedings of the Exeter Symposium July 1982. IAHS Publ. no. 138, 1982.

Choquette, Y., Lavigne, P., Nadeau, M., Ducharm, P., Martin, J., Houdayer, A., and Rogoza, J.: GMON, a new sensor for snow water equivalent via gamma monitoring, in: Proceedings Whistler 2008 International Snow Science Workshop, 21-27 September 2008, Whistler, B.C., pp. 802-807, https://arc.lib.montana.edu/snow-science/objects/P_8132.pdf, 2008.

de Mendonça, R. R. S., Braga, C. R., Echer, E., Lago, A. D., Munakata, K., Kuwabara, T., Kozai, M., Kato, C., Rockenbach, M., Schuch, N. J., Jassar, H. K. A., Sharma, M. M., Tokumaru, M., Duldig, M. L., Humble, J. E., Evenson, P., and Sabbah, I.: The temperature effect in secondary cosmic rays (muons) observed at the ground: Analysis of the global muon detector network data, The Astrophysical Journal, 830, 88, https://doi.org/10.3847/0004-637x/830/2/88, 2016.

Desilets, D., Zreda, M., and Ferré, T. P.: Nature's neutron probe: Land surface hydrology at an elusive scale with cosmic rays, Water Resources Research, 46, https://doi.org/10.1029/2009WR008726, 2010.

Ganeva, M., Peglow, S., Hippler, R., Berkova, M., and Yanke, V.: Seasonal variations of the muon flux seen by muon telescope MuSTAnG, Journal of Physics: Conference Series, 409, 012 242, https://doi.org/10.1088/1742-6596/409/1/012242, 2013.

GLAMOS: The Swiss Glaciers 2017/18-2018/19, Glaciological Reports No 139-140, in: Yearbooks of the Cryospheric Commission of the Swiss Academy of Sciences (SCNAT), edited by Bauder, A., Huss, M., and Linsbauer, A., p. 155p, published since 1964 by VAW/ ETH Zurich, https://doi.org/10.18752/glrep139-140, 2020.

Gugerli, R.: Towards improved spatio-temporal snow observations in glacierized high mountain regions by a multi-sensor approach, Ph.D. thesis, University of Fribourg, Switzerland, 2021.

Gugerli, R., Salzmann, N., Huss, M., and Desilets, D.: Continuous and autonomous snow water equivalent measurements by a cosmic ray sensor on an alpine glacier, The Cryosphere, 13, 3413-3434, https://doi.org/10.5194/tc-2019-106, 2019.

Howat, I. M., De La Peña, S., Desilets, D., and Womack, G.: Autonomous ice sheet surface mass balance measurements from cosmic rays, The Cryosphere, 12, 2099-2108, https://doi.org/10.5194/tc-12-2099-2018, 2018.

Kinar, N. J. and Pomeroy, J. W.: Measurement of the physical properties of the snowpack, Reviews of Geophysics, 53, 481-544, https://doi.org/10.1002/2015RG000481.Received, 2015.

Kodama, M.: Continuous Monitoring of Snow Water Equivalent Using Cosmic-Ray Neutrons, Cold Regions Science and Technology, 3, 295-303, https://doi.org/10.1016/0165-232X(80)90036-1, 1980.

Kodama, M., Kawasaki, S., and Wada, M.: A cosmic-ray snow gauge, The International Journal Of Applied Radiation And Isotopes, 26, 774-775, https://doi.org/10.1016/0020-708X(75)90138-6, 1975.

Kodama, M., Nakai, K., Kawasaki, S., and Wada, M.: An application of cosmic-ray neutron measurements to the determination of the snow-water equivalent, Journal of Hydrology, 41, 85-92, 1979.

MeteoSchweiz: Klimabulletin February 2021, Tech. rep., Zürich, https://www.meteoschweiz.admin.ch/content/dam/meteoswiss/de/ service-und-publikationen/Publikationen/doc/202102_d.pdf, last access 2 August 2021, 2021.

Nitu, R., Roulet, Y., Wolff, M., Earle, M., Reverdin, A., Smith, C., Kochendorfer, J., Morin, S., Rasmussen, R., Wong, K., Alastrué, J., Arnold, L., Baker, B., Buisan, S., Collado, J. L., Colli, M., Collins, B., Gaydos, A., Hannula, H.-R., Hoover, J., Joe, P., Kontu, A., Laine, T., Lanza, L., Lanzinger, E., Lee, G. W., Lejeune, Y., Leppänen, L., Mekis, E., Panel, J., Poikonen, A., Ryu, S., Sabatini, F., Theriault, J., Yang, D., 
https://doi.org/10.5194/tc-2021-277

Preprint. Discussion started: 7 September 2021

(C) Author(s) 2021. CC BY 4.0 License.

(c) (1)

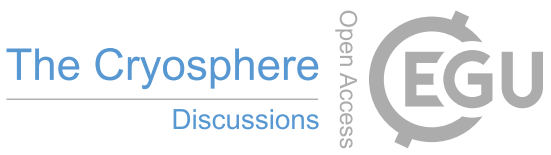

Genthon, C., van den Heuvel, F., Hirasawa, N., Konishi, H., Nishimura, K., and Senese, A.: WMO Solid Precipitation Intercomparison Experiment (SPICE) (2012-2015), Tech. Rep. 131, Geneva, 2018.

Osterhuber, R., Gehrke, F., and Condreva, K.: Snowpack snow water equivalent measurement using the attenuation of cosmic gamma radiation, in: Western Snow Conference, Snowbird, Utah, USA, 1998.

Rasmussen, R., Baker, B., Kochendorfer, J., Meyers, T., Landolt, S., Fischer, A. P., Black, J., Thériault, J. M., Kucera, P., Gochis, D., Smith, C., Nitu, R., Hall, M., Ikeda, K., and Gutmann, E.: How well are we measuring snow: The NOAA/FAA/NCAR winter precipitation test bed, Bulletin of the American Meteorological Society, 93, 811-829, https://doi.org/10.1175/BAMS-D-11-00052.1, 2012.

Riádigos, I., García-Castro, D., González-Díaz, D., and Pérez-Muñuzuri, V.: Atmospheric Temperature Effect in Secondary Cosmic Rays Observed With a 2 m2 Ground-Based tRPC Detector, Earth and Space Science, 7, e2020EA001 131, https://doi.org//10.1029/2020EA001131, 2020.

Schattan, P., Baroni, G., Oswald, S. E., Schöber, J., Fey, C., Kormann, C., Huttenlau, M., and Achleitner, S.: Continuous monitoring of snowpack dynamics in alpine terrain by aboveground neutron sensing, Water Resources Research, 53, 3615-3634, https://doi.org/10.1002/2016WR020234, 2017.

Wada, M., Kodama, M., and Kawasaki, Y.: Method of determining the water equivalent depth of snowfall using neutrons of cosmic rays, united States Patent, US 4,047,042, 1977. 